\title{
Heparina de alto peso molecular. Uma alternativa nas operações com circulação extracorpórea: estudo experimental
}

\author{
Roberto CATANI*, Ayrton BERTINI Jr.*, Clodualdo J. N. PESSA*, Walter J. GOMES*, \\ Dayse M. LOURENÇO*, Helena B. NADER*, Carl P. DIETRICH*, João Nelson R. BRANCO*, \\ Enio BUFFOLO*
}

RBCCV 44205-543

\begin{abstract}
Catani R, Bertini Jr. A, Pessa C J N, Gomes W J, Lourenço D M, Nader H B, Dietrich C B, Branco J N R, Buffolo E. - Heparina de alto peso molecular. Uma alternativa nas operações com circulação extracorpórea: estudo experimental. Rev Bras Cir Cardiovasc 2001; 16(2): 160-170.
\end{abstract}

RESUMO: Introdução: As síndromes hemorrágicas no intra e pós-operatório de operações com circulação extracorpórea (CEC) constituem motivo de preocupação e, parte delas, pode ser atribuída à heparina não fracionada (HNF), droga indispensável e, até hoje, insubstituível nesse tipo de procedimento. Alguns autores consideram a ação anticoagulante da HNF como o principal responsável pelo sangramento e investem em drogas antifibrinolíticas ou que alteram a atividade plaquetária para tentar substituí-la. Toda HNF contém frações de heparina de baixo peso molecular (HBPM), não neutralizáveis pela protamina, que, em doses elevadas, e/ou em pacientes heparino-sensíveis, podem causar vasoplegia e aumento no sangramento pós-operatório em operações com CEC.

Material e Métodos: Isolamos uma heparina de alto peso molecular (HAPM - peso modal de 25.000 Daltons), com $11 \%$ de frações de HBPM (< 7.000 Daltons), para experiências "in vitro" e "in vivo", e comparamos com HNF (peso modal de 15.000 Daltons), com $21 \%$ de frações de HBPM.

Resultados: A atividade específica anticoagulante, por massa, foi superior quando comparada com a HNF tanto "in vitro", $273 \mathrm{ui} / \mathrm{mg}$ contra $181 \mathrm{ui} / \mathrm{mg}$ e TTPA mais elevado nas várias diluições, como "in vivo", em cães, durante CEC, comprovado pelo TCA, TTPA e heparinemia. A vida média da HNF foi de 60 minutos e acima de 90 minutos para a HAPM, na situação de experimentação.

Conclusão: Acreditamos que esta experiência, inédita na literatura indexada, nos habilite ao uso da HAPM, em seres humanos, para averiguação da sua melhor neutralização pela protamina e menor incidência de hemorragia.

DESCRITORES: Heparina, uso terapêutico. Anticoagulantes, uso terapêutico. Circulação extracorpórea. Anticoagulantes, química. Heparina, química.

\footnotetext{
Trabalho realizado na Escola Paulista de Medicina - Universidade Federal de São Paulo. São Paulo, SP, Brasil. Apresentado ao $27^{\circ}$ Congresso Nacional de Cirurgia Cardíaca. Rio de Janeiro, 23 a 25 de março de 2000.

* Da Disciplina de Cirurgia Cardiovascular da Escola Paulista de Medicina - Universidade Federal de São Paulo.

Endereço para correspondência: Roberto Catani. Rua Nicolau de Souza Queiroz, 297, apto. 74. Paraíso. São Paulo, SP, Brasil. CEP 01525-001.

Tel: (11) 9196-4682. e-mail: cardiovascular.dcir@epm.br
} 
Catani R, Bertini Jr. A, Pessa C J N, Gomes W J, Lourenço D M, Nader H B, Dietrich C B, Branco J N R - Buffolo E. Heparina de alto peso molecular. Uma alternativa nas operações com circulação extracorpórea: estudo experimental. Rev Bras Cir Cardiovasc 2001; 16(2): 160-170.

\section{INTRODUÇÃO}

A heparina, formada principalmente nos mastócitos, encontrada em vários animais invertebrados, e na maioria dos vertebrados, foi descoberta, acidentalmente, por sua atividade anticoagulante (1), a qual ocorre pela potencialização da propriedade natural isolada da antitrombina e seu co-fator ${ }^{(2)}$. O aperfeiçoamento do método de extração e purificação sob a forma cristalina de sal de bário (3) permitiu obter-se quantidades suficientes para utilização na clínica médica.

Desde o advento da circulação extracorpórea (CEC), em maio de1953 (4), a heparina apresentase como substância indispensável nesse tipo de procedimento, apesar de várias tentativas experimentais de substituí-la por drogas sintéticas ou enzimas com ação anticoagulante ${ }^{(5-8)} \mathrm{e}$, até mesmo, por heparina de baixo peso molecular (HBPM) $(9,10)$, sem sucesso. Este fato, em particular, chamou-nos a atenção o que justifica detalharmos um pouco mais o embasamento teórico da tese que defenderemos neste trabalho.

Hoje sabemos que o produto comercialmente utilizado (heparina não fracionada - HNF) constituise, na realidade, de uma mistura de várias espécies de heparinas com pesos moleculares diferentes, cuja mediana desses pesos, isto é, o peso modal, se encontra próximo de 12.000 Daltons para as bovinas e 15.000 Daltons para as suínas. A heparina é um polissacarídeo ácido pertencente à família química dos glicosaminoglicanos ${ }^{(11)}$ e sua estrutura foi elucidada através da degradação enzimática seqüencial da molécula ${ }^{(12)}$. Uma molécula com 2.000 Daltons seria a menor heparina conhecida (Figura 1) (13). Essas moléculas interligadas lado a lado formam espécies com atividade diferente e pesos moleculares cada vez maiores, chegando a heparinas nativas com 60.000 a 100.000 Daltons (14). TAKAHASHI (15) separou 63 tipos diferentes de heparinas com peso molecular variável entre 2.000 e 32.000 Daltons e atividade anticoagulante variável entre 28 e 348 ui/mg. A ação anticoagulante é própria das heparinas de alto peso molecular (HAPM > 15.000 Daltons). São características da heparina de elevada ação anticoagulante: 1) possuir alto peso molecular $(16,17) ; 2)$ ter grande afinidade pela antitrombina (18); 3) conter alta densidade e homogeneidade de cargas aniônicas (15).

As heparinas de baixo peso molecular (HBPM $<6.000$ Daltons) têm atividade antitrombótica, aceleram a inibição do fator $\mathrm{Xa}{ }^{(19)}$ e interagem com as plaquetas ${ }^{(20)}$, sem atuarem na trombina ${ }^{(19)}$. Desde o modo original de se preparar fragmentos de heparina com ácido ascórbico, usando-se sulfato de cobre como catalizador (21), muitos outros foram introduzidos.

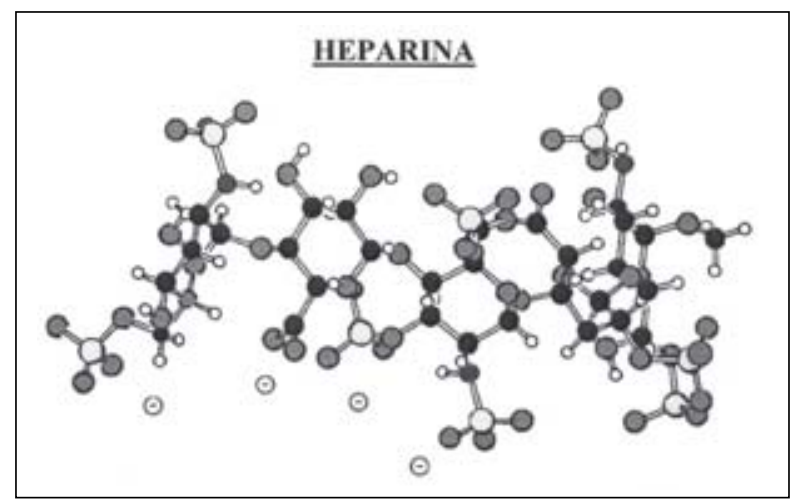

Fig. 1 - Ilustração - Estrutura molecular - Pentassacarídeo com afinidade à antitrombina III, mostrando as cargas negativas de 3 grupos de sulfato e 1 de carboxilato (RAGAZZI et al., 1987)

As heparinas comerciais de baixo peso molecular são isoladas pelo fracionamento através de vários métodos ${ }^{(22,23)}$. Este avanço no arsenal terapêutico trouxe comprovado e indiscutível benefício no tratamento de síndromes coronarianas agudas, nos pacientes portadores de próteses mecânicas e que precisam retroca, na hemodiálise, na prevenção de trombose venosa profunda, etc. O sucesso clínico e comercial se evidencia pelo gasto, no ano de 1999, de 2,7 bilhões de dólares com HBPM contra 300 milhões de dólares com HNF (24). No entanto, tentativas experimentais com HBPM, em CEC, foram desastrosas. Doses altas foram necessárias, muito acima das utilizadas clinicamente $(2.700$ a $4.500 \mathrm{ui} / \mathrm{Kg}$ ), e, mesmo não anticoagulados, apresentaram sangramento intenso no pós-operatório (9). Experimentos semelhantes em cães mostraram também grande sangramento havendo uma morte por hemorragia (10). Em ambos os trabalhos $(9,10)$ não foi utilizada neutralização com protamina.

Vários estudos sugerem que as HBPM têm atividade anti-hemostática por se ligarem a receptor molecular da miosina presente na musculatura lisa dos pequenos vasos inibindo sua contratilidade e aumentando o sangramento(25-34). Na circulação extracorpórea utilizamos, temporariamente, altas doses de HNF. Sabe-se que a protamina não neutraliza as HBPM, as quais, circulando, poderiam causar vasoplegia. De encontro ao exposto, experiências "in vitro" e "in vivo" mostraram que, por sua semelhança, a molécula de trifosfato de adenosina tópica desaloja a HBPM do músculo liso, permitindo o retorno de sua função vasoconstritora, tanto em animais ${ }^{(28)}$, como no pós-operatório de operação com circulação extracorpórea, diminuindo o sangramento (35-37).

Indica-se a suspensão de drogas antiagregantes plaquetárias, alguns dias antes da operação, para 
Catani R, Bertini Jr. A, Pessa C J N, Gomes W J, Lourenço D M, Nader H B, Dietrich C B, Branco J N R - Buffolo E. Heparina de alto peso molecular. Uma alternativa nas operações com circulação extracorpórea: estudo experimental. Rev Bras Cir Cardiovasc $2001 ; 16(2): 160-170$

se evitar hemorragia. Nossa premissa teórica é que, à semelhança disto, seria bom para operação cardíaca com CEC se conseguíssemos obter comercialmente uma HAPM, com elevada ação anticoagulante, totalmente neutralizável pela protamina, sem os efeitos colaterais das frações de HBPM com ação anti-hemostática.

Este estudo tem por objetivo avaliar se uma HAPM pode ser confeccionada e utilizada em CEC com segurança.

\section{MATERIAL E MÉTODOS}

Foram utilizadas e comparadas duas heparinas, posteriormente neutralizadas com protamina. HNF, LIQUEMINE ROCHE lote 706003619. HAPM, confeccionada pelos laboratórios OPOCRIN e NOVARTIS especialmente para este trabalho, obtida por fracionamento em acetato de bário a diferentes temperaturas ${ }^{(38)}$. Protamina $1000 \mathrm{ROCHE}$ lote 709594159.

\section{Procedimentos Experimentais "in vitro"}

Determinação da mediana do peso molecular das duas heparinas (peso modal) pela microeletroforese em gel de poliacrilamida (39).

Determinação do percentual dos vários pesos moleculares, separados em 12 bandas, das duas heparinas, pela eletrofocalização em gel de poliacrilamida $(40)$.

Comparação da ação anticoagulante de doses progressivas das duas heparinas, em uma mistura de plasmas frescos, pelo teste tempo de tromboplastina parcial ativado (TTPA), caulim e silimat (41). Utilizou-se um "pool" de 11 plasmas frescos, de doadores humanos voluntários, pobres em plaquetas, obtido por punção única venosa periférica, anticoagulado com citrato de sódio $100 \mathrm{mM}$, na proporção 1:9 (volume/volume) e centrifugado a 3.000 r.p.m., por 15 minutos, à temperatura ambiente. A heparinização com doses progressivas das duas heparinas permitiu amostras de plasma com as seguintes concentrações: zero, um, dois e meio, cinco, dez, 20, 50 e $75 \mathrm{mg} / \mathrm{ml}$ (microgramas de heparina por mililitro de plasma fresco).

Determinação da atividade anticoagulante das duas heparinas pelo método U.S.P. (The United States Pharmacopeia) ${ }^{(42) .}$

\section{Procedimentos Experimentais "in vivo"}

Comparação da ação anticoagulante das duas heparinas, na dose de $4 \mathrm{mg} / \mathrm{kg}$, em dois grupos de
5 cães, adultos, sem raça definida, em igual estado de nutrição, por sorteio aleatório, nove do sexo masculino e um do feminino, com peso variando de 9,2 a $23,2 \mathrm{~kg}$, procedentes do biotério central da Escola Paulista de Medicina, de acordo com os princípios experimentais éticos e humanos, submetidos à circulação extracorpórea com oxigenador de bolhas Macchi (pediátrico ou infantil) carregado com $500 \mathrm{ml}$ de Ringer lactato, com coleta de sangue antes da heparinização, cinco minutos, 30', 60', 90' após heparinização e dez minutos após neutralização com protamina. Realização das seguintes curvas, com a média dos resultados: tempo de coagulação ativado (43), tempo de tromboplastina parcial ativado (41), heparinemia ${ }^{(44,45)}$, plaquetas, hematócrito e calculada a vida média das duas heparinas (38).

\section{Método Estatístico}

Para a análise dos resultados foram utilizados testes não paramétricos em decorrência da natureza das distribuições das variáveis estudadas. Foram aplicados os seguintes testes:

1- análise de variância por postos de Friedman (46) com o objetivo de comparar os valores da heparinemia, separadamente para cada tipo de heparina, nos diversos períodos de tempo de heparinização. Quando mostrou diferença significante, esta análise foi complementada pelo teste de comparações múltiplas(47);

2- teste de Mann-Whitney para duas amostras independentes (46) quando comparou-se os valores da heparinemia observados com os métodos de Caulim e Silimat. O mesmo teste foi aplicado para comparar cada um dos métodos, as duas heparinas estudadas.

Em todos os testes fixou-se em 0,05 ou $5 \%$ (alfa $\leq 0,05)$ o nível para rejeição da hipótese de nulidade e assinalando-se com um asterisco $\left({ }^{*}\right)$ os valores significantes.

\section{RESULTADOS}

O peso modal, pela microeletroforese, foi de 15.000 Daltons para a HNF e de 25.000 Daltons para a HAPM.

$\mathrm{Na}$ Tabela 1 apresentamos o resultado do peso molecular de doze frações das duas heparinas, pela eletrofocalização, e o porcentual encontrado.

$\mathrm{Na}$ Tabela 2 apresentamos os resultados dos TTPAs realizados pelo caulim e silimat, nas diferentes diluições usadas no "pool" de plasmas frescos humanos. 

peso molecular. Uma alternativa nas operações com circulação extracorpórea: estudo experimental. Rev Bras Cir Cardiovasc $2001 ; 16(2): 160-170$.

\section{TABELA 1}

\begin{tabular}{cccr}
\hline \multicolumn{4}{c}{$\begin{array}{c}\text { RESULTADO DOS PESOS MOLECULARES DE DOZE } \\
\text { FRAÇOES DAS DUAS HEPARINAS. } \\
\text { EXPRESSO EM \% }\end{array}$} \\
\hline FRAÇÕES & $\begin{array}{c}\text { PM } \\
\text { KDA }\end{array}$ & $\begin{array}{r}\text { HNF } \\
\%\end{array}$ & $\begin{array}{r}\text { HAPM } \\
\%\end{array}$ \\
\hline 1 & $21-25$ & 14,2 & 37,5 \\
2 & $18-20$ & 8,2 & 9,7 \\
3 & $15-17$ & 28,1 & 24,4 \\
4 & $12-14$ & 23,3 & 1,9 \\
5 & $10-11$ & 2,7 & 1,5 \\
6 & $8-9$ & 1,7 & 12,9 \\
7 & $7-8$ & 0,8 & 1,1 \\
8 & 6 & 6,5 & 3,1 \\
9 & 5 & 8,2 & 4,6 \\
\hline 10 & 4 & 0,9 & 0,7 \\
11 & 3 & 1,2 & 1,3 \\
12 & 2 & 4,2 & 1,3 \\
\hline
\end{tabular}

$\mathrm{KDA}=$ Kilodaltons
TABELA 2

RESULTADO DO TEMPO DE TROMBOPLASTINA PARCIAL ATIVADO, EM "POOL" DE PLASMA FRESCO HUMANO, COM DOSES PROGRESSIVAS DE HEPARINA NÃO FRACIONADA E DE ALTO PESO MOLECULAR. EXPRESSO PELA RELACÃO ENTRE O TTPA DO PLASMA HEPARINIZADO E O TTPA DO PLASMA SEM HEPARINA

\section{GRAU DE HEPARINIZAÇÃO \\ HNF \\ HAPM}

$\mu \mathrm{g} / \mathrm{ml}$ CAULIM SILIMAT CAULIM SILIMAT

\begin{tabular}{rrrrr}
\hline 0,00 & 1,00 & 1,00 & 1,00 & 1,00 \\
\hline 1,00 & 1,21 & 1,07 & 1,44 & 1,19 \\
\hline 2,50 & 2,33 & 1,97 & 2,92 & 3,16 \\
\hline 5,00 & 4,07 & 5,94 & 6,34 & $*$ \\
\hline 10,00 & 6,29 & - & 10,46 & - \\
\hline 20,00 & 13,50 & - & 31,35 & - \\
\hline 50,00 & 62,84 & - & 70,28 & - \\
\hline 75,00 & $*$ & & $*$ & - \\
\hline
\end{tabular}

* incoagulável - não realizado
A atividade anticoagulante, pelo método U.S.P., foi de 181 ui/mg para a HNF e de 273 ui/mg para a HAPM.

Nos Gráficos 1 a 5 apresentamos as curvas dos TCAs, TTPAs, heparinemias, plaquetas e hematócritos, das duas heparinas, respectivamente, durante experimentação "in vivo".

Nas Tabelas 3 e 4 apresentamos o resultado da heparinemia, em cada amostra, das duas heparinas, durante experimentação "in vivo".

$\mathrm{Na}$ Tabela 5 apresentamos o cálculo da vida média das duas heparinas.

\section{TABELA 3}

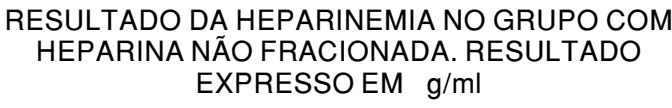

RESULTADO DA HEPARINEMIA NO GRUPO COM HEPARINA NÃO FRACIONADA. RESULTADO EXPRESSO EM $\mathrm{g} / \mathrm{ml}$

\begin{tabular}{lrrrrrr}
\hline $\begin{array}{l}\text { TEMPO } \\
\text { HEPARINA }\end{array}$ & $\mathbf{5}$ & $\mathbf{3 0}$ & $\mathbf{6 0}$ & $\mathbf{9 0}$ & $\mathbf{1 0}$ ' PÓs \\
\hline A1 & 0 & 12,33 & 3,43 & 5,95 & 0,62 & 0,52 \\
B1 & 0 & 3,69 & 1,76 & 1,31 & 0,94 & 0,31 \\
C1 & 0 & 3,76 & 1,51 & 0,95 & 0,64 & 0,00 \\
\hline D1 & 0 & 6,06 & 3,71 & 2,70 & 4,27 & 0,18 \\
\hline E1 & 0 & 14,50 & 1,16 & 4,65 & 1,16 & 0,00 \\
\hline MÉDIA & 0 & 8,07 & 2,31 & 3,11 & 1,53 & 0,12 \\
\hline
\end{tabular}

TABELA 4

RESULTADO DA HEPARINEMIA NO GRUPO COM HEPARINA DE ALTO PESO MOLECULAR. RESULTADO EXPRESSO EM $\mathrm{g} / \mathrm{ml}$

\begin{tabular}{|c|c|c|c|c|c|c|}
\hline \multicolumn{2}{|c|}{$\begin{array}{l}\text { TEMPO PRÉ } \\
\text { HEPARINA }\end{array}$} & \multirow{2}{*}{$\begin{array}{l}\mathbf{5}^{\prime} \\
7,46\end{array}$} & \multirow{2}{*}{$\begin{array}{c}30^{\prime} \\
7,10\end{array}$} & \multirow{2}{*}{$\begin{array}{l}\frac{60^{\prime}}{} \\
5,42\end{array}$} & \multirow{2}{*}{$\begin{array}{l}90^{\prime} \\
1,53\end{array}$} & \multirow{2}{*}{$\begin{array}{r}10^{\prime} \mathrm{POS} \\
0,00\end{array}$} \\
\hline A2 & 0,00 & & & & & \\
\hline B2 & 0,00 & 33,00 & 26,30 & 29,25 & 34,00 & 0,87 \\
\hline C2 & 0,00 & 13,00 & 3,85 & 3,83 & 1,54 & 0,57 \\
\hline $\mathrm{D} 2$ & 0,00 & 15,00 & 7,99 & 4,47 & 4,62 & 0,98 \\
\hline E2 & 0,00 & 14,30 & 9,91 & 7,51 & 5,45 & 0,76 \\
\hline MÉ & 0,00 & 16,55 & 11,03 & 10,10 & 9,43 & 0,64 \\
\hline
\end{tabular}


Catani R, Bertini Jr. A, Pessa C J N, Gomes W J, Lourenço D M, Nader H B, Dietrich C B, Branco J N R - Buffolo E. Heparina de alto peso molecular. Uma alternativa nas operações com circulação extracorpórea: estudo experimental. Rev Bras Cir Cardiovasc $2001 ; 16(2): 160-170$.

TABELA 5

CÁLCULO DA VIDA MÉDIA DAS DUAS HEPARINAS, EM CÃES, DURANTE CIRCULAÇÃO EXTRACORPÓREA

\begin{tabular}{lcccc}
\hline \multicolumn{5}{c}{ HEPARINA NÃO FRACIONADA } \\
\hline $\begin{array}{l}\text { TEMPO } \\
\text { HEPARINA }\end{array}$ & 5 & $30^{\prime}$ & $60^{\prime}$ & $90^{\prime}$ \\
\hline V.E.P.H. & 8,07 & 5,24 & 5,75 & 7,56 \\
V.O. & 8,07 & 2,31 & 3,11 & 1,53 \\
$\%$ DO ESP. & $100 \%$ & $44 \%$ & $54 \%$ & $20 \%$ \\
\hline
\end{tabular}

HEPARINA DE ALTO PESO MOLECULAR

\begin{tabular}{lcccr}
\hline $\begin{array}{l}\text { TEMPO } \\
\text { HEPARINA }\end{array}$ & 5 & $300^{\prime}$ & 60 & $90^{\prime}$ \\
\hline V.E.P.H. & 16,55 & 12,81 & 13,40 & 13,99 \\
\hline V.O. & 16,55 & 11,03 & 10,10 & 9,43 \\
\% DO ESP. & $100 \%$ & $86 \%$ & $75 \%$ & $67 \%$ \\
\hline
\end{tabular}

V.E.P.H. = Valor esperado pela hemodiluição. EM g/ml; V.O. = valor obtido em $\mathrm{g} / \mathrm{ml}$.; \% DO ESP. = porcentual do valor esperado.

\section{COMENTÁRIOS}

A HAPM por nós obtida tem peso modal de 25.000 Daltons, contra 15.000 Daltons da HNF, com a qual foi comparada no estudo.

Pela eletrofocalização, pudemos identificar que nossa heparina tem $71,6 \%$ das frações $>15.000$ Daltons, mas não é totalmente destituída de HBPM, contém $11 \%$ de frações < 7.000 Daltons contra $21 \%$ para a HNF (Tabela 1).

\section{Análise da Anticoagulação "in vitro"}

A relação do TTPA, nos dois métodos realizados em plasma humano fresco, mostrou elevação mais acentuada, com uma mesma dose, para a HAPM, conferindo a esta um poder maior de anticoagulação do que a HNF (Tabela 2). Isto foi confirmado pela determinação da atividade anticoagulante das duas heparinas, pelo método U.S.P., quando constatamos que a HAPM tem 1,5 vez mais eficiência do que a HNF (273 ui/mg contra $181 \mathrm{ui} / \mathrm{mg})$.

\section{Análise da Anticoagulação "in vivo"}

A análise dos TCAs, TTPAs e heparinemias nos dois grupos, durante a circulação extracorpórea, confirmou que a atividade anticoagulante foi maior na HAPM do que na HNF (Gráficos 1, 2 e 3).

Aos cinco minutos após a administração de heparina obtivemos, nos dois grupos, o máximo de concentração plasmática (Tabelas 3 e 4). A heparinemia individual de cada animal variou muito além do que se pode imaginar para erro de dose ou método diagnóstico (Tabelas 3 e 4), fato também observado em humanos $(29,48)$ e que permanece inexplicável. Seria a sensibilidade individual à heparina a grande responsável? ${ }^{(31,32)}$.

A neutralização com protamina foi mais eficiente no grupo com HAPM, talvez por conter menos frações de baixo peso molecular. No experimento, $6,78 \%$ da heparina não foi neutralizada no grupo da HAPM contra $7,84 \%$ na HNF. A implicação clínica disto é que as HBPM ficam circulando (48), saem pelo dreno ${ }^{(36)}$ e podem ter sido as responsáveis pelo maior sangramento nos casos em que foram utilizadas em circulação extracorpórea $(9,10)$, e a causa do sangramento "inexplicável", pós-operatório, maior, nos casos descritos com altas doses de $\operatorname{HNF}(49,50)$.

Durante a CEC, a plaquetopenia foi semelhante nos dois grupos (Gráfico 4). O mesmo ocorreu com a hemodiluição, calculada através da volemia estimada pelo peso e a queda do hematócrito (Gráfico 5). Partindo-se do princípio que aos cinco minutos temos o máximo de heparinemia, isto é, $100 \%$, podemos calcular o valor esperado pela hemodiluição aos 30, 60 e 90 minutos. De posse do valor esperado e o obtido em cada tempo, calculamos a atenuação da heparina em função do tempo (vida média). A vida média da HNF, na dose usada, foi de 60 minutos e mais de 90 minutos para a HAPM (Tabela 5).

Durante a experimentação, a perda sangüínea foi desprezível nos dois grupos.

O tamanho da amostra foi pequeno e não permitiu detectar diferença significativa entre as duas heparinas, fato que, a princípio, não foi o objetivo do trabalho.

\section{Considerações Finais}

Foi por sua propriedade anticoagulante que a heparina se firmou no arsenal terapêutico, permanecendo, até hoje, insubstituível nas operações com CEC. No pós-operatório dessas operações, parte das síndromes hemorrágicas pode ser atribuída à heparinização. Acreditando que o sangramento ocorria pela anticoagulação sangüínea ${ }^{(18)}$, várias tentativas foram preconizadas, desde o acerto de doses menores, controladas rigorosamente com o TCA $(49,50)$, até o uso de drogas sintéticas. Estamos 


\section{GRÁFICO 1 \\ MÉDIA DAS DETERMINAÇÕES DO TCA NOS GRUPOS COM AS \\ DUAS HEPARINAS, NO PERÍODO DE TEMPO DA AVALIAÇÃO}

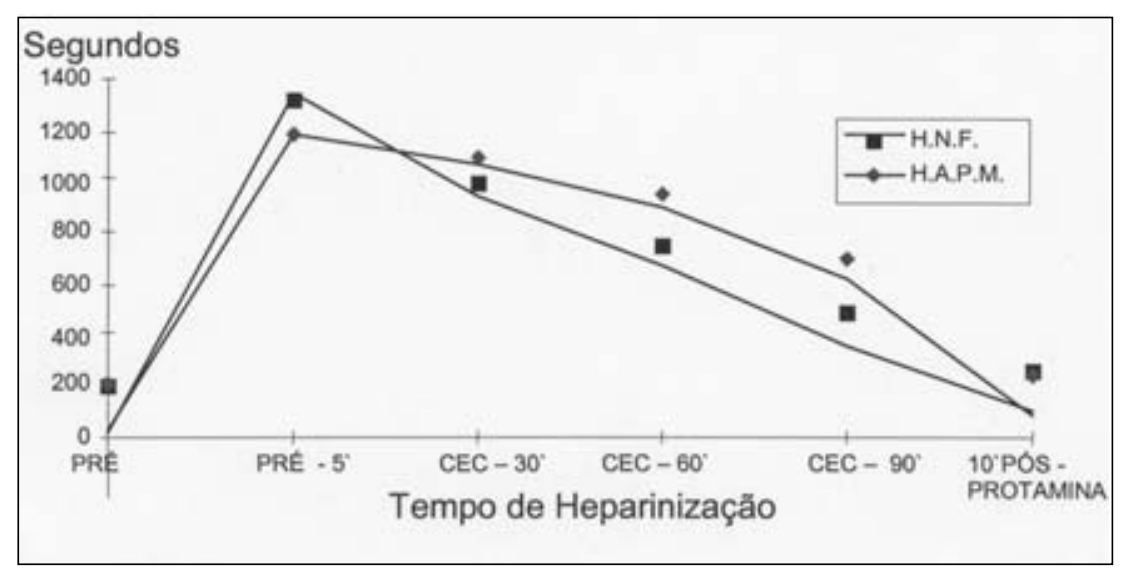

\section{GRÁFICO 2}

MÉDIA DAS DETERMINAÇÕES DO TTPA NOS GRUPOS COM AS

DUAS HEPARINAS, NO PERÍODO DE TEMPO DA AVALIAÇÃO.

EXPRESSO PELA RELAÇÃO ENTRE O TTPA DO PLASMA

HEPARINIZADO E O TTPA DO PLASMA SEM HEPARINA

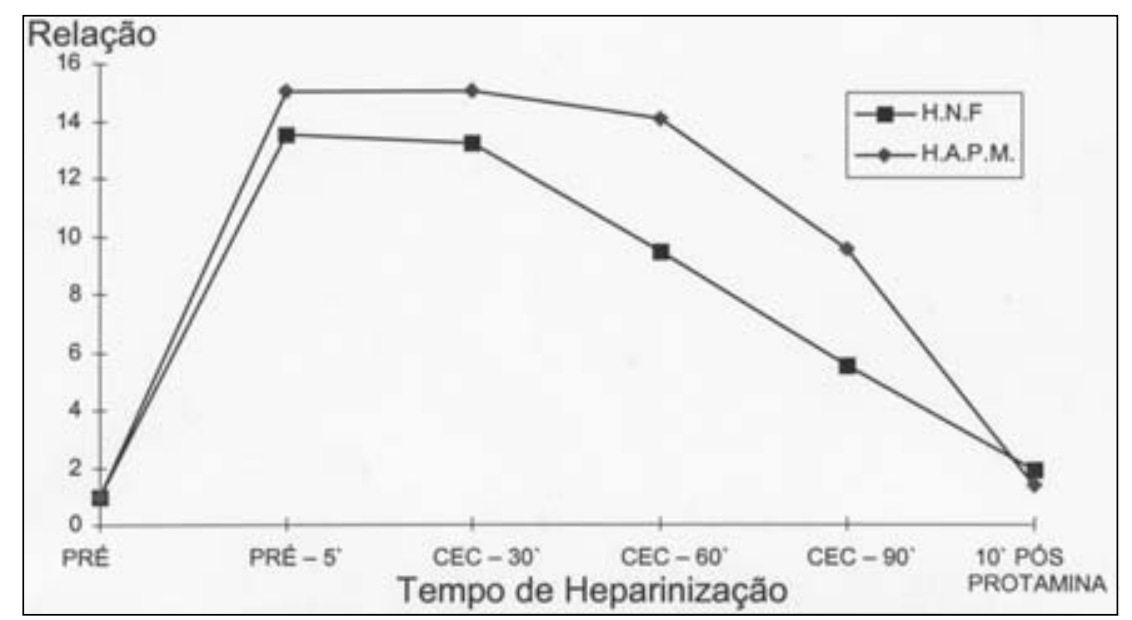

convencidos de que a neutralização da ação anticoagulante com protamina é importante para diminuir a drenagem pós-operatória ${ }^{(51)}$. No entanto, a experiência frustante com HBPM em CEC nos deixou mais convictos de que a anticoagulação não é a única responsável pelo sangramento, reforçando uma antiga linha de pesquisa de nossa Instituição que valoriza a ação vascular da heparina $(28,29)$. Com o empenho do prof. Dietrich obtivemos uma HAPM cujo peso modal é de 25.000 Daltons. Como não encontramos trabalhos indexados, clínicos ou experimentais, com esse tipo de heparina, resolvemos compará-la, "in vitro" e "in vivo", em cães, com HNF (peso modal de 15.000 Daltons).

A questão é: pode a HAPM substituir a HNF nas operações com CEC, com segurança?

A HAPM tem ação anticoagulante maior do que a HNF, por massa. É possível se obter uma HAPM pelo método de acetato de bário a diversas tempe- 

peso molecular. Uma alternativa nas operações com circulação extracorpórea: estudo experimental. Rev Bras Cir Cardiovasc $2001 ; 16(2): 160-170$

\section{GRÁFICO 3}

MÉDIA DA HEPARINEMIA NOS GRUPOS COM AS DUAS

HEPARINAS, NO PERÍODO DE TEMPO DA AVALIAÇÃO

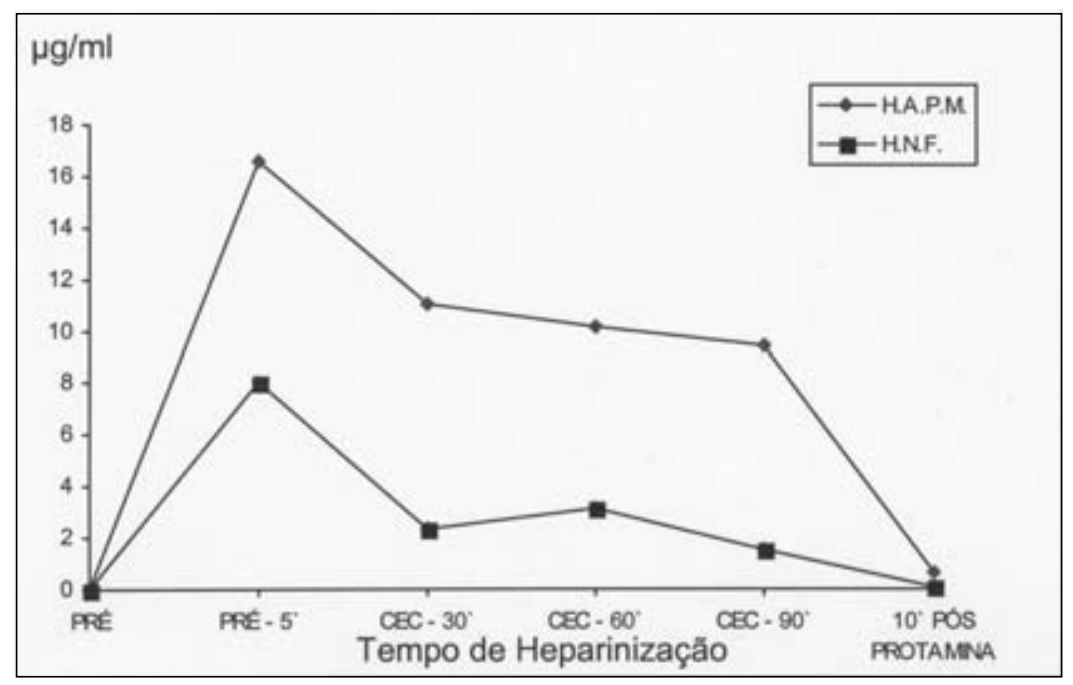

GRÁFICO 4

MÉDIA DA CONTAGEM DAS PLAQUETAS NOS GRUPOS COM AS DUAS HEPARINAS, NO PERÍODO DE TEMPO DA AVALIAÇÃO

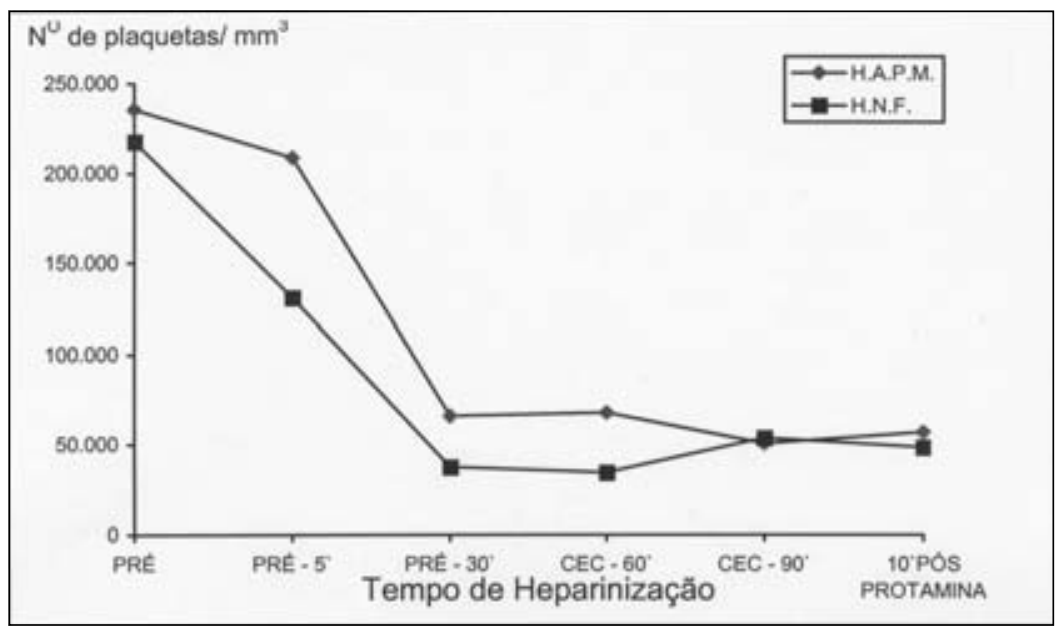

raturas. A cromatografia de troca iônica em DEAEcelulose ${ }^{(15)}$ e outros métodos poderão retirar ainda mais HBPM da HNF.

Acreditamos que este trabalho experimental nos habilite a propor novos estudos para a utilização de uma HAPM em CEC, em humanos.

Estima-se que no ano de 1999, mundialmente, cerca de 1.000 .000 de cirurgias foram realizadas com CEC, movimentando mais de 24 milhões de dólares só com heparinização. Seguramente, esta cifra poderá motivar interesses empresariais para o desenvolvimento de diversos tipos de HAPM que poderão oferecer menor sangramento nas operações com CEC e permitir melhor neutralização pela protamina. 
Catani R, Bertini Jr. A, Pessa C J N, Gomes W J, Lourenço D M, Nader H B, Dietrich C B, Branco J N R - Buffolo E. Heparina de alto peso molecular. Uma alternativa nas operações com circulação extracorpórea: estudo experimental. Rev Bras Cir CardiovasC $2001 ; 16(2): 160-170$.

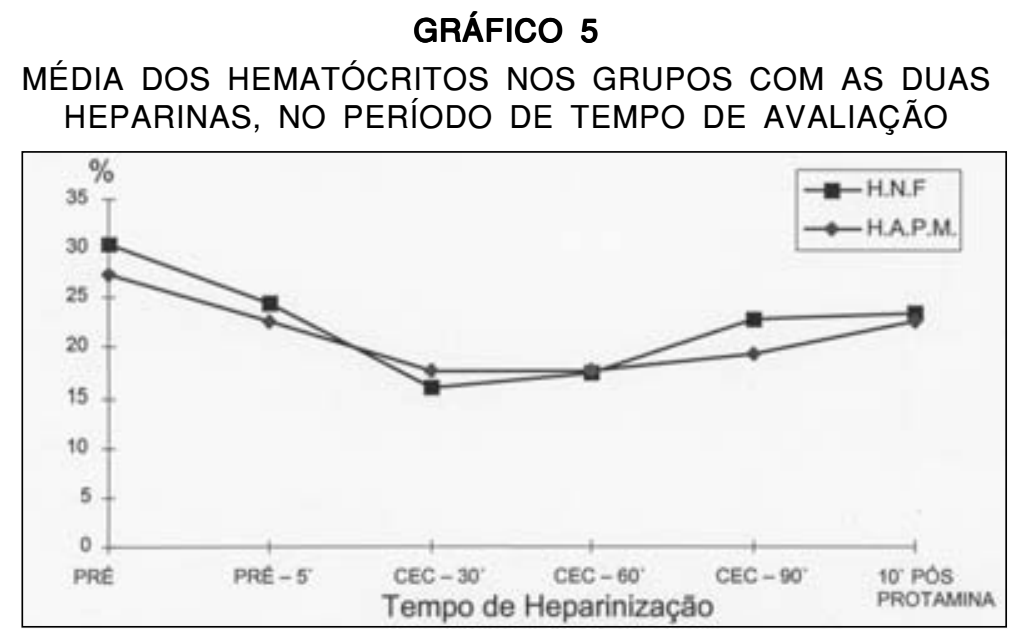

\section{CONCLUSÕES}

\section{$\mathrm{Na}$ situação da experimentação:}

1. A HAPM "in vitro" tem ação anticoagulante em plasma humano fresco e, na mesma dose (em peso), altera mais o TTPA do que a HNF.

2. A HAPM por nós obtida tem $273 \mathrm{ui} / \mathrm{mg}$ de atividade anticoagulante enquanto a HNF usada tem $181 \mathrm{ui} / \mathrm{mg}$.
3. Durante a CEC a HAPM, com a mesma dose em $\mathrm{mg} / \mathrm{kg}$ de peso do animal, alterou mais o TCA e 0 TTPA, e por mais tempo, do que a HNF.

4. A heparinemia 5 ' após a administração intravenosa, em cães, foi maior para a HAPM do que para a HNF.

5. A vida média da HNF foi de 60' e mais $90^{\prime}$ para a HAPM.

6. Nenhuma das duas heparinas foi totalmente neutralizada pelo cloridrato de protamina.

Catani R, Bertini Jr. A, Pessa C J N, Gomes W J, Lourenço D M, Nader H B, Dietrich C B, Branco J N R, Buffolo E. - High molecular weight heparin. An alternative in extracorporeal circulation surgery: an experimental study. Rev Bras Cir Cardiovasc 2001; 16(2): 160-170.

ABSTRACTS: Introduction: In surgical procedures, with cardiopulmonary bypass, hemorrhagic syndromes during and after pump constitute a major concern and in a great number of cases they are heparin-related, a substance still without substitute. Most authors point out the anticoagulant action of heparin as the main problem in bleeding situations and research is developing on antifibrinolytic or platelet-like- drugs to try to substitute for usual heparin. Experience with low-molecular weight heparin, without anticoagulant properties, in cardiopulmonary bypass, was disastrous. High dosage was accompanied by high tube drainage suggesting that postoperative bleeding does not happen just because of the anticoagulant effect of heparin.

Material and Methods: Believing in the vascular component of hemostasis and that low-molecular weight heparin non-neutralized by protamin is responsible for the paralysis of small vessels during and after cardiopulmonary bypass surgery, we isolated a high-molecular weight heparin ( modal weight of 25.000 Daltons) to be tested "in vitro" and "in vivo".

Results: Its specific anticoagulant activity, by mass, was superior to usual heparin ( modal weight of 15.000 Daltons) "in vitro" (273 ui/mg against $181 \mathrm{ui} / \mathrm{mg}$, respectively) as "in vivo", in dogs, utilizing cardiopulmonary bypass and measuring its activity by activated clotting time, APTT and heparin blood levels. In the experimental laboratory the half-life of usual heparin was of 60 minutes, while for highmolecular weight heparin was above 90 minutes.

Conclusion: We believe that this unprecedented experience will lead to its future use in "anima nobile" to further test its neutralization by protamin as well as to confirm the decreased prevalence of bleeding phenomena with its use.

DESCRIPTORS: Heparin, therapeutic use. Anticoagulants, therapeutic use. Extracorporeal circulation. Anticoagulants, chemistry. Heparin, chemistry. 

peso molecular. Uma alternativa nas operações com circulação extracorpórea: estudo experimental. Rev Bras Cir Cardiovasc $2001 ; 16(2): 160-170$.

\section{AGRADECIMENTO}

A todos que colaboraram na confeç̧ão deste trabalho e não foram citados como co-autores, em especial, o Sr. Pietro Bianchini que fracionou a HAPM no laboratório OPOCRIN, a Dra. Marila Ferraz que possibilitou a diluição, ampolagem e esterilização da HAPM com o padrão NOVARTIS e o Dr. José Carlos Silva de Andrade que gentilmente revisou o texto.

\section{REFERÊNCIAS BIBLIOGRÁFICAS}

1 Mc Lean J - The discovery of heparin. Circulation 1959; 19: 75-8.

2 Jordan R E, Oosta G M, Gardner W T, Rosenberg R D - The kinetics of hemostatic enzyme-antithrombin interactions in the presence of low molecular weight heparin. J Biol Chem 1980; 255: 10081-90.

3 Charles A F \& Scott D A - Studies on heparin IV. Observations on the chemistry of heparin. Biochem J 1936; 30: 1927-31.

4 Gibbon Jr. J - Application of a mechanical heart and lung apparatus to cardiac surgery. Minn Med 1954; 37: 171-80.

5 Takano H, Miyamoto $\mathrm{Y}$, Kaneko $\mathrm{M}$ et al. - Experimental study on heparin-free cardiopulmonary bypass using nafamostat mesilate (FUT-175) and heparin-coated circuit. Nippon Kyobu Geka Gakkai Zasshi 1993; 41: 2166-73.

6 Chomiak P N, Walenga J M, Koza M J, Reilly TM, Turlapathy P, Pifarre R - Investigation of a thrombin inhibitor peptide as an alternative to heparin in cardiopulmonary bypass surgery. Circulation 1993; 88(5 pt 2): II407-12.

7 Doherty D C, Ortel T L, De Bruijn N, Greenberg CS, Van Trigt P 3rd - "Heparin-free" cardiopulmonary bypass: first reported use of heparinoid (ORG. 10.172) to provide anticoagulation for cardiopulmonary bypass. Anesthesiology 1990; 73: 562-5.

8 Zulys $\mathrm{V}$ J, Teasdale S J, Michel E R et al. -
Ancrod (ARVIN) as an alternative to heparin anticoagulation for cardiopulmonary bypass. Anesthesiology 1989; 71: 870-7.

9 Massonet-Castel S, Pelissier E, Dreyfus G et al. - Low-molecular-weight heparin in extracorporeal circulation. Lancet 1984; 1: 1182-3. [Letter]

10 Touchot B, Laborde F, Dum F et al. - Use of low molecular weight heparin CY222 during cardiopulmonary bypass: experimental study and clinical application. Perfusion 1986; 1: 99-103.

11 Jeanloz R W - The nomenclature of mucopolysaccharides. Arthritis Rheum 1960; 3: 233-7.

12 Dietrich C P - Novel heparin degradation products. isolation and characterization of novel disaccharides and oligosaccharides produced from heparin by bacterial degradation. Biochem $J$ 1968; 108: 647-54.

13 Ragazzi M, Ferro D R, Perry B et al. Conformation of the pentasaccharide corresponding to the binding site of heparin antithrombin III. Carbohydr Res 1987; 165: $\mathrm{C} 1-5$

14 Robinson H C, Horner A A, Höök M, Ogren S, Lindahl $U$ - A proteoglycan form of heparin and its degradation to single-chain molecules. J Biol Chem 1978; 253: 6687-93.

15 Takahashi H K - Isolamento e caracterização de espécies moleculares de heparina com diferentes graus de afinidade por antitrombina III: correlação com atividades farmacológicas [Tese. Doutorado] São Paulo: Escola Paulista de Medicina - UNIFESP, 1993. 173p.

16 McDuffie N M, Dietrich C P, Nader H B Electrofocusing of heparin: fractionation of heparin into 21 components distinguishable from other acidic mucopolysaccharides. Biopolymers 1975; 14: 1473-86. 

peso molecular. Uma alternativa nas operações com circulação extracorpórea: estudo experimental. Rev Bras Cir CardiovasC 2001; 16(2): 160-170.

17 Holmer E, Söderberg K, Bergqvist D, Lindahl $U$ - Heparin and its low molecular weight derivates: anticoagulant and antithrombotic properties. Haemostasis 1986; 16(suppl2): 1-7.

18 Schwartz B S - Heparin: what is it? How does it work? Clin Cardiol 1990; 13 (4 suppl 6): VI 12-5.

19 Holmer E - Low molecular weight heparin. In: Lane D A \& Lindahl U, eds. - Heparin, chemical and biological properties: clinical applications. London: Edward Arnold Publishers, 1989: 575-96.

20 Salzman E W, Rosenberg R D, Smith M H, Lindon J N, Favreau L - Effect of heparin and heparin fractions on platelet aggregation. J Clin Invest 1980; 65: 64-73.

21 Dietrich C P, Nader H B, Mcduffie N N Electrofocusing of heparin: presence of 21 monomeric and dimeric molecular species in heparin preparations. An Acad Bras Cienc 1975; 47: 301-9.

22 Lasker S E \& Stivala S S - Physicochemical studies of fractionated bovine heparin. I. Some dilute solution properties. Arch Biochem Biophys 1966; 115: 360-72.

23 Bianchini P, Osima B, Parma B, Nader H B, Dietrich $C \mathrm{P}-\mathrm{Pharmacological}$ activities of heparins obtained from different tissues: enrichment of heparin fractions with high lipoprotein lipase, antihemolytic, and anticoagulant activities by molecular sieving and antithrombin III affinity chromatography. J Pharmacol Exp Therap 1982; 220: 406-10.

24 Fareed J, Hoppensteadt D A, Bick R L - An update on heparins at the beginning of the new millennium. Semin Thromb Hemost 2000; 26(suppl.1): 5-21.

25 Cruz W O - The significance of a smooth muscle component in hemostasis. Proc Soc Exp Biol Med 1965; 119: 876-83.
26 Dietrich C P \& Magalhães J R - Effect on bleeding time of topical application of anticoagulants. An Acad Bras Cienc 1965; 37: 141-4.

27 Magalhães J R, Unglert C V, Damiani H, Nogueira Filho $\mathrm{J}$ - Anti-hemostatic action of heparin: relationship between topical and intravenous application. Acta Physiol Lat Am 1971; 21: 209-15.

28 Cruz W O \& Dietrich C P - Antihemostatic effect of heparin counteracted by adenosine thriphosphate. Proc Soc Exp Biol Med 1967; 126: $420-6$.

29 Dietrich C P, Tersariol I L S, Da Silva R G, Bianchini P, Nader H B - Dependence of the C-6 sulfate of the glicosamine moiety and 1-4 glycosidic linkage of heparin disacharides for production of hemorrhage: reversal of the antihemostatic activity of heparin and their fragments by adenosine triphosphate and myosin. Semin Thromb Hemost 1991; 17(suppl 1): 65-73.

30 Tersariol I L, Dietrich C P, Nader H B Interaction of heparin with myosin ATPase: possible involvement with the hemorrhagic activity and a correlation with antithrombin III high affinity - heparin molecules. Thromb Res 1992; 68: 247-58.

31 Tanezini C A - Características farmacológicas da ação tópica anti-hemostática da heparina em ratos, coelhos e humanos [Tese. Mestrado] São Paulo: Escola Paulista de Medicina - UNIFESP, 1976. 116 p.

32 Magalhães J R - Hemostase cutânea em humanos [Tese. Doutorado] São Paulo: Escola Paulista de Medicina - UNIFESP, 1983. 115p.

33 Nader H B \& Dietrich C P - Effect of heparin sulfate fractions on coagulation and hemostasis. Proc Soc Exp Biol Med 1974; 146: $504-8$.

34 Ireland H, Rylance P B, Kesteven P - Heparin as an anticoagulant during extracorporeal 

peso molecular. Uma alternativa nas operações com circulação extracorpórea: estudo experimental. Rev Bras Cir Cardiovasc $2001 ; 16(2): 160-170$

circulation. In: Lane D A \& Lindahl U, eds. - Heparin, chemical and biological properties: clinical applications. London: Edwards Arnold Publishers, 1989: 549-74.

35 Garcia Jr. H V - Estudo da ação tópica do trifosfato de adenosina no sangramento produzido pela heparina em cirurgia com utilização de circulação extracorpórea [Tese. Doutorado] São Paulo: Escola Paulista de Medicina - UNIFESP, 1994. 81p.

36 Garcia Jr. H V, Buffolo E, Nader H B, Dietrich C P - ATP reduces blood loss produced by heparin in cardiopulmonary bypass operations. Ann Thorac Surg 1994; 57: 956-9.

37 Dietrich C P, Shinjo S K, Moraes F A et al. - Structural features and bleeding activity of commercial low molecular weight heparins: neutralization by ATP and protamine. Semin Thromb Hemost 1999; 25(suppl.3): 43-50.

38 Catani $\mathrm{R}$ - Heparina de alto peso molecular: uma alternativa para cirurgias com circulação extracorpórea. Estudo experimental [Tese. Mestrado] São Paulo: Escola Paulista de Medicina - UNIFESP, 1994. 134p.

39 Dietrich C P \& Nader H B - Fractionation and properties of four heparitin sulfates from beef lungs tissues: isolation and partial characterization of a homogeneous species of heparitin sulfate. Biochim Biophys Acta 1974; 343: 34-44.

40 Nader H B, McDuffie N M, Dietrich C P Heparin fractionation by electrofocusing: presence of 21 components of different molecular weights. Biochem Biophys Res Commun 1974; 57: 488-93.

41 Proctor R S \& Rapaport S I - The partial thromboplastin time with kaolin: a simple screening test for first stage plasma clotting factor deficiencies. Am J Clin Pathol 1961; 36: 212-9.

42 Bianchini $\mathrm{P}$, Osima B, Parma B, Dietrich C P, Takahashi H K, Nader H B - Structural studies and "in vivo" and "in vitro" pharmacological activities of heparin fractions and fragments prepared by chemical and enzymic depolimerization. Thromb Res 1985; 40: 49-58.

43 Hattersley P G - Activated coagulation time of whole blood. JAMA 1966; 196: 436-40.

44 Jaques $L$ B, Ballieux $R$ E, Dietrich $C P$, Kavanagh L W - A microelectrophoresis method for heparin. Can J Physiol Pharmacol 1968; 46: 351-60.

45 Dietrich C P \& Dietrich S M - Electrophoretic behaviour of acidic mucopolysaccharides in diamine buffers. Anal Biochem 1976; 70: 645-7.

46 Siegel S - Estatística não paramétrica. México: Ed. Trilhas, 1975. p.346.

47 Hollander M \& Wolfe D A - Nonparametric statistical methods. New York: John Willy \& Sons, 1973. p.503.

48 Moraes Neto F R - Dosagem da heparina em cirurgia cardíaca com circulação extracorpórea [Tese. Mestrado] São Paulo: Escola Paulista de Medicina - UNIFESP, 1994. 50p.

49 Gravlee G P, Haddon W S, Rothberger H K et al. - Heparin dosing and monitoring for cardiopulmonary bypass: a comparison of techniques with measurement of subclinical plasma coagulation. J Thorac Cardiovasc Surg 1990; 99: 518-27.

50 Cardoso P F, Yamazaki F, Keshavjee S et al - A reevaluation of heparin requirements for cardiopulmonary bypass. J Thorac Cardiovasc Surg 1991; 101: 153-60.

51 Buffolo E, Forte V, Gallucci C et al. - Pacientes submetidos a cirurgia cardíaca com neutralização ou não da heparinização intra - operatória. In: Anais do XXV Congresso Brasileiro de Cardiologia, Belo Horizonte, MG. Sociedade Brasileira de Cardiologia, 1969: p.83. 